\title{
Seamless care, do it well or not at all
}

\section{J. W. Foppe van Mil ${ }^{1}$}

Published online: 21 November 2019

(c) Springer Nature Switzerland AG 2019

To avoid errors and harm to patients, proper transfer of patient data is important. Many local, regional and national structures have been created that should secure patient safety across transitions in care, the so called seamless care. Apart from historical patient data, especially the continuity of drug treatment is an important element of the transition. The drug treatment should be based on a valid list of medicines that a patient needs at the given moment.

But any system is as good as its components, and identifying the weakest link is, or should be, part of the system analysis, also for continuity of care. A recent short stay in a well-run hospital revealed unexpected flaws in the system that are worth discussing.

Patient A was admitted to a large academic hospital, with a decrease in kidney function and low magnesium after cisplatinum/etoposide treatment. After the nurses, who asked for the patients' drug use and entered it into the computer, the physician did his own intake and checked and discussed the current medication (as in the computer) and time of administration with the patient. A DOAC was replaced by nadroparine. The physician then confirmed in the hospital system that this medication would be continued after the adaptation discussed (replacing the DOAC) and the physician activated the medication in the hospital system so that patient should receive his medication on the ward. Then another nurse, doing the admissions on the ward, once again checked the list with the patient, and the list still seemed correct.

After admission on the ward, a pharmacy assistant also discussed the home medicine use with the patient, based on a list that was obtained from a national database-in which some older prn medication also appeared. This list is taken from a database to which all community pharmacies in the Netherlands are linked. This dispensed

J. W. Foppe van Mil

jwfvmil@ijcp-editor.org

1 Margrietlaan 1, 9471CT Zuidlaren, The Netherlands medication list did contain some minor inaccuracies (some medication was not stopped), that were corrected after talking with the patient and then it also reflected the real actual drug use picture of the patient. For privacy reasons, the pharmacy assistant did not have access to the hospital records of the patient.

So, at the end of the admission, four different health professionals were informed about the medication of the patient. If the pharmacy assistant somehow also reconciled her records with the hospital records, and looked at optimisation, is not clear. In this given case such a reconciliation step did not seem to have taken place.

\section{The events}

The first day it was decided by the ward physician (which was yet another physician from the one who did the intake) that candesartan should be stopped, due to the lower renal function and an acceptable blood pressure, and the patient indeed did not receive the medication again. That afternoon patient was informed that Gastilox (a combination of algeldrate with magnesium oxide) could not be dispensed by the pharmacy because they did not have it on stock. The patient was not aware of his need to have this medication and had not asked for it, or mentioned it. But it later appeared that the oral algeldrate/ magnesium was started according to a certain protocol by a physician, because the patient had low serum-magnesium. As the pharmacy did not have the medication Gastilox on stock, that evening the patient received a chewing tablet with magnesiumoxide.

On the second day, lercanidipine was missing from the medication, apparently removed from the medication list, actor and reason unclear to patient and nursing staff. At the daily round, the ward physician decided to stop the metformin, and regulate the diabetes with insulin. That evening, however, the metformin was still offered and the patient refused the medication (the nurse did not know that the medication was discontinued). The diabetes was 
regulated with insulins by protocol by the nurses, based on blood glucose readings. The magnesium chewing tablet, which was given the first night as supplement, was not given on the second day, because it was now lacking on the dispensing list. The third day the patient again received no lercanidipine, the metformin was manually withheld by the nurses, who now knew about the stop, but now the patient suddenly received two tablets of paracetamol at breakfast, although there was no pain reported by the patient.

We will not go into further detail about varying administration times, or clinical relevance because these are less important in the context.

\section{The weakest link}

In the above case, all technical facilities and protocols for medication reconciliation seem to be in place. The continuity of care standard was adhered to, yet the patient did not receive what he should receive, and alterations took place in the actual medication list without the patient or nurses being informed. A quick root cause analysis brought all errors back to the weakest link, the human behaviour. It is clear in this case the humans involved were of the best of intentions, but at the same time the weakest link in the communication about the medication changes to patient and staff. Table 1 gives the medication errors, and the potential reasons and actors involved.

The term reconciliation appears a lot in literature, but often as another term for medication review. To ensure seamless care when patients are being transferred, all resources, from the hospital and the pharmacy, need to be reconciled into a final list of care related information, including the medicines that the patient should have in the hospital. In the above mentioned case, all information was available, but apparently not being reconciled. All the information provided and obtained in the beginning, was very complete, but not reconciled, and there also was no optimisation effort. Then the alterations in the hospital list were implemented without information, or not implemented.

\section{Discussion}

\section{Reconciliation}

In this case, already at the outset of the hospitalisation, different people compiled different drug lists but these lists were not always reconciled. Although understandable that this happens, it must be clear from the beginning what the use of the different lists is and how they can be merged. It should also be clear which list is to be the real reflection of the proposed drug-use of the patient in the hospital and must be communicated as such. From efficiency and patient satisfaction perspectives, you would like to have one person performing, and being fully responsible for, the medication reconciliation. From a seamless care perspective, resources from the patient, pharmacy and the hospital need to be reconciled into a final list of medicines the patient should have. In the context of this commentary, we will call this the rootmedicine list, a list of medicines that the patient should have when in hospital according to the pharmacists and physicians.

\section{Access rights and identification}

The root-medicine list in a hospital must be accessible to all involved in the care of the patient (if the patient has given consent to share his data) and to the patient himself. This list must be amended and optimised after regular medication review by pharmacists and the daily rounds of the physicians. All changes in this root-medicine list should be traceable by name, date, and reason for change. Treatment changes must be communicated to the staff and the patient. A responsible person for the treatment must always be in-house and reachable for the staff and patient. In this hospital system, changes in the list were not (and could perhaps not be) commented, but it seems essential, if a drug is stopped, started or altered, that it is easily traceable why, when and by whom.

Table 1 Medication errors and probable causes

\begin{tabular}{|c|c|c|c|c|}
\hline 1 & Gastilox cannot be dispensed & $\begin{array}{l}\text { Low serum-magnesium. Physician ordered } \\
\text { Mg supplement during chemotherapy but } \\
\text { did not tell patient or nurse }\end{array}$ & $\begin{array}{l}\text { Pharmacy responded to nurse, not to physi- } \\
\text { cian. }\end{array}$ & Human error \\
\hline 2 & Lercanidipine not dispensed & Not prepared and dispensed & $\begin{array}{l}\text { Medicine deactivated/removed from list? } \\
\text { Why? }\end{array}$ & Human error? \\
\hline 3 & Metformin dispensed & Not stopped timely in system & Physician probably forgot & Human error \\
\hline 4 & Paracetamol dispensed & Prn overlooked & Nurse did not see. Patient had no pain & Human error \\
\hline 5 & Magnesium only dispensed once & Not ordered. See 1 & Physician did not tell patient or nurse & Human error \\
\hline
\end{tabular}




\section{Authorisation}

Changes in the root-list of medicines should ideally be double checked. This means that at least two professionals must be authorised to delete, add or change medicines in the root list, preferably in combination. Thus it must not be possible to change the root-medicine list in the spur of the moment, not only to protect the patient but also to make sure that the why, when and by whom questions are always answered and documented.

\section{Reverse process}

Once the patient is being discharged, a reverse process should take place. Patient and care providers in the new setting should know exactly what medicines (and care) patients should receive; they should have a medicine list provided by the hospital on which the actual medication use is clear. And another list explaining what has happened and why changes were implemented after the setting initially changed (e.g. the hospital admission). In the setting discussed, this also is not properly taken care of, potentially leading to errors in treatment at home.

\section{Role of the patient}

Ideally, patients also should monitor the root-medicines list, and be co-responsible. But especially in the hospital setting, most patients are older and often confused, or perhaps even unconscious. Patients and their family will fully trust the professionals around them. It is unethical to count on the patient as responsible party for his continuity of care under such stressful circumstances as a hospitalisation. But patients should have access to their medication list in hospital, if they wish.

\section{Conclusion}

The technicalities of seamless care seem to be well implemented in many hospitals. However, when looking at what patients receive, and what they should receive, there seem to be obvious flaws in the system in the given case. Most of the causes can be found in insufficient communication, and the human and professional interaction between the different professionals therefore seems to be the missing link.

Seamless care and medication reconciliation will not work if healthcare professionals are focused on generating medication lists instead of collaborating with each other to keep the medication list accurate. But it must also be kept in mind that the patient (or his carer) is the only constant factor in healthcare. Empowering patients to have access to their medication list during hospitalization could also help in mitigating errors.

Acknowledgements The author wishis to acknowledge the assistance of Dr. F. Karapinar, hospital pharmacist, in the writing of this editorial.

Publisher's Note Springer Nature remains neutral with regard to jurisdictional claims in published maps and institutional affiliations. 